\title{
Transplante autógeno de canino superior incluso com acompanhamento de 13 anos
}

13-year follow-up of impacted upper canine autogenous transplantation

Transplante autogeno de canino superio incluido con seguimiento de 13-años

José Carlos Garcia de MENDONÇA ${ }^{1}$

Muryllo Eduardo Sales dos SANTOS ${ }^{2}$

Maria José Bazualdo SORUCO²

Ellen Cristina GAETTI-JARDIM ${ }^{3}$

${ }^{1}$ Professor Associado, Faculdade de Odontologia da Universidade Federal de Mato Grosso do Sul (FAODO), Universidade Federal do Mato Grosso do Sul (UFMS) 79070-900 Campo Grande - MS, Brasil

${ }_{2}^{2}$ Programa de Residência em Cirurgia e Traumatologia Bucomaxilofacial, Faculdade de Odontologia da Universidade Federal de Mato Grosso do Sul (FAODO) Universidade Federal do Mato Grosso do Sul (UFMS) 79070-900 Campo Grande - MS, Brasil

${ }^{3}$ Professora Doutora da Faculdade de Odontologia da Universidade Federal de Mato Grosso do Sul (FAODO)

Universidade Federal do Mato Grosso do Sul (UFMS) 79070-900 Campo Grande - MS, Brasil

\section{Resumo}

O canino superior é um dos dentes que apresentam grande predisposição a impacção, a etiologia dessa problemática é incerta, uma das hipóteses levantadas é devido a ser um dos últimos dentes a erupcionar na arcada. O transplante dental é uma alternativa de tratamento em casos de dentes impactados cujo posicionamento na arcada dentaria com tracionamento ortodôntico não seja possível. Também em casos onde a exodontia seguida de reabilitação com implantes não é uma opção. O objetivo deste trabalho é relatar um caso clínico de autotransplante dental de um canino superior incluso, com rizogênese completa em um paciente de 17 anos. O caso foi acompanhado por 13 anos, mostrando um bom prognóstico, e indicando o transplante autógeno como uma ótima solução clínica, estética e social.

Descritores: Dente não Erupcionado; Terapêutica; Diagnóstico.

\section{Abstract}

The upper canine is one of the teeth that has a great predisposition to impaction, the etiology of this problem is uncertain, one of the hypotheses raised is due to the upper canine being one of the last teeth to erupt in the arch. Tooth transplantation is an alternative treatment in cases of impacted teeth whose positioning in the dental arch with orthodontic traction is not possible. Also in cases where extraction followed by implants rehabilitation is not an option. The aim of this work is to report a clinical case of tooth autotransplantation of an impacted upper canine, with complete rhizogenesis in a 17-year-old patient. The case was followed for 13 years, showing a good prognosis, and indicating autogenous transplantation as a great clinical, aesthetic and social solution.

Descriptors: Tooth, Unerupted; Therapy; Diagnosis.

\section{Resumen}

El canino superior es uno de los dientes con mayor propensión a impactaciones, la etiología de esta complicación es incierta, entre las hipótesis sugeridas, asumimos que se da por ser uno de los últimos dientes en erupcionar en el arco. El trasplante dental es un tratamiento alternativo en casos de dientes impactados cuyo reposicionamiento en el arco dental a través de tracción ortodóntica no sea posible. Así como en casos donde la extracción seguida de rehabilitación con implantes no es considerada una alternativa. El objetivo de este trabajo es reportar un caso clínico de un autotrasplante dental de canino superior incluso, con rizogénesis completa en una paciente de 17 años. Fue dado seguimiento al caso durante 13 años, mostrando un buen pronóstico, e sugiriendo el trasplante autógeno como una óptima solución clínica, estética y social.

Descriptores: Diente no Erupcionado; Terapéutica; Diagnóstico.

\section{INTRODUÇÃO}

O transplante dental autógeno é utilizado na odontologia desde $1950^{1}$. Consiste na transposição de um órgão dental para uma nova localização nas arcadas dentárias sendo para um alvéolo natural, previamente ocupado por um elemento dental ou um alvéolo confeccionado cirurgicamente para receber o germe dentário a ser transferido ${ }^{1-3}$.

Essa modalidade de tratamento é utilizada para a substituição de elementos dentários comprometidos devido à presença de periodontopatias, alterações endodônticas, destruição coronária causada por fraturas ou lesões cariosas, que impedem um tratamento odontológico satisfatório do dente afetado sendo indicada a exodontia ${ }^{4}$. Outra indicação se dá em casos de dentes inclusos ou impactados onde o dente é transposto em posição na arcada dentaria ${ }^{2}$.

A impacção de elementos dentários pode ser influenciada pelo posicionamento anormal do elemento dentário, patologias, ausência de espaço no arco dentário para seu percurso normal de erupção, dilaceração radicular, diâmetro da coroa ou da raiz do dente e fatores sistêmicos individuais de cada paciente ${ }^{5,6}$.

Caninos superiores estão entre os dentes que apresentam maior predisposição a impacção, sendo o gênero feminino o mais afetado nessa condição clínica ${ }^{6}$. A etiologia que justifica essa frequência não é bem esclarecida, uma das causas apontadas é o fato de ser um dos últimos dentes a erupcionar ${ }^{5,7}$.

A manutenção do elemento dentário sem qualquer tipo de tratamento não é recomendada devido a possibilidade de ocorrência de patologias relacionadas com o capuz coronário e possibilidade de reabsorção radicular de dentes adjacentes como incisivos, laterais e prémolares permanentes ${ }^{5,7}$. O tratamento de caninos impactados em palato varia desde tracionamento ortodôntico, exodontia do elemento dentário ou transplante autógeno do órgão dental ${ }^{5,6}$.

O transplante autógeno apresenta maior sucesso em paciente com idade até 17 anos ou elementos dentários com rizogênese dentaria 
incompleta, devido a vantagem de revascularização da polpa do germe dentário ${ }^{4}$. Essa técnica também pode ser bem executada em dentes com rizogênese completa associado a tratamento endodôntico, apresentando sucesso no tratamento ${ }^{2,8}$.

A técnica deve ser sugerida a pacientes que não tenham interesse em tratamento ortodôntico e apresentem espaço na arcada dentaria compatível com o diâmetro da coroa do dente impactado, ou pacientes sem condições financeiras para reabilitação com implante na região após exodontia de canino incluso ${ }^{1}$.

$O$ relato de caso exposto tem como objetivo descrever o acompanhamento clínico e radiográfico durante 13 anos de um transplante autógeno de canino com rizogênese completa, procedimento em que foi realizado exodontia do elemento incluso em palato e transplantado em alvéolo cirurgicamente confeccionado.

\section{CASO CLÍNICO}

Paciente do sexo feminino, 17 anos, leucoderma, procurou atendimento com queixa estética na região do canino superior esquerdo, devido à grande espaço presente.

Ao exame radiográfico observou-se canino incluso por palatino com rizogênese completa. Havia boa estrutura óssea e espaço compatível para se fazer o transplante dentário para o lugar em que o dente ocuparia na arcada dentária (Figura1).

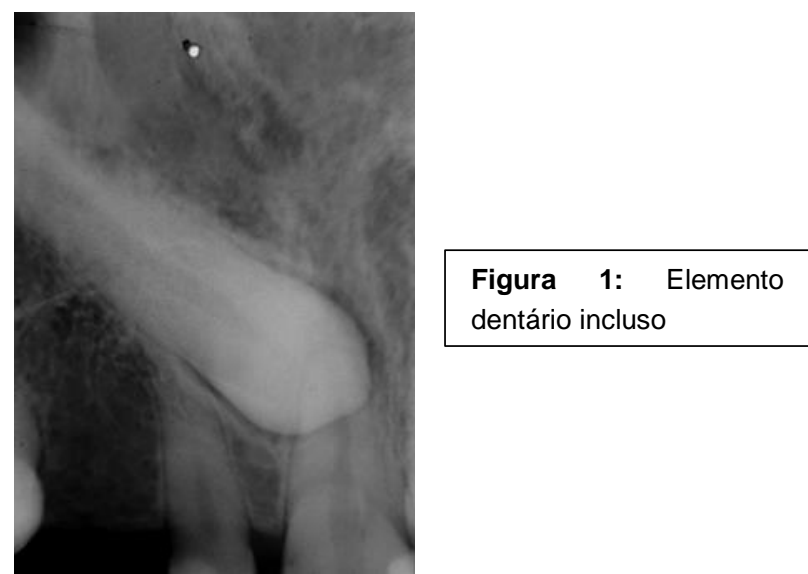

Avaliou-se clínica e radiograficamente a compatibilidade do dente impactado com a região que receberia o transplante, no sentido mesio-distal e vestíbulo-lingualmente.

Considerando-se a queixa estética da paciente e suas limitações financeiras, optou-se pelo transplante dental autógeno, com confecção do alvéolo cirúrgico.

O procedimento cirúrgico foi realizado sob anestesia local por bloqueio dos nervos nasopalatino, alveolar superior anterior e médio, com lidocaína à 2\% com adrenalina 1:50000. Realizou-se a incisão intrasulcular em região palatina de incisivo central esquerdo até distal de segundo pré-molar superior ipsilateral, descolamento e afastamento mucoperiosteal da região. Seguiu-se de ostectomia ampla e cuidadosa, com broca cirúrgica adequada, sob irrigação com soro fisiológico 0,9\%, para expor o elemento dentário incluso. Uma ostectomia ampla tem como objetivo facilitar a remoção do dente e evitar o traumatismo do ligamento periodontal. Prosseguindo com a luxação delicada do elemento dentário com auxílio de alavanca seldin reta, sem a remoção do mesmo. Realizou-se incisão e descolamento da fibormucosa vestibular, expondo o rebordo alveolar adjacente. Preparou-se por meio de broca cirúrgica, sob irrigação copiosa com soro fisiológico $0,9 \%$, uma cavidade óssea com a forma e dimensão do alvéolo para receber o dente incluso, preservando máximo da lâmina vestibular. Seguiu-se a remoção do elemento incluso. Observou-se uma dilaceração radicular importante, que impedia a inserção do elemento no novo alvéolo cirúrgico. Procedeu-se a apicectomia da região, com alicate ortodôntico de corte, para inserção em alvéolo. Após o dente transplantado estar bem adaptado no novo alvéolo, fixou-se o mesmo, em infraoclusão, através de amarria com fio de aço $\mathrm{N}^{\circ} 0$. Finalizando com sutura interdental dos tecidos moles com fio de poliamida 4.0.

No pós-operatório imediato, foi prescrito Amoxicilina 500mg, 01 capsula de 08/08h, durante 07 dias; Ibuprofeno $600 \mathrm{mg}, 01$ comprimido de 08/08h, por 03 dias, Dipirona $500 \mathrm{mg} / \mathrm{ml}, 30$ gotas de 06/06h se necessário e Digluconato de Clorexidina $0,12 \%$, para bochechos com de 12/12h, por 07 dias. Paciente foi orientada a manter dieta pastosa e não morder na região operada por 60 dias. No acompanhamento inicial a paciente não apresentou sinais de infecção na região transplantada ou qualquer outra queixa.

Aos 15 dias de pós-operatório realizouse abertura coronária, extirpação da polpa e obturação temporária do canal com pasta de hidróxido de cálcio, com intuito de diminuir a reabsorção radicular e o selamento biológico apical $^{9-12}$.

No retorno pós-operatório de 30 dias realizou-se a remoção da contenção. O elemento dentário apresentava-se sem mobilidade, com reinserção gengival adequada e sem sinais flogísticos. Observou-se clínica e radiograficamente que a unidade transplantada estava adaptada ao novo alvéolo e a reparação óssea e do ligamento periodontal estavam ocorrendo normalmente.

Aos 60 dias pós-operatórios realizou-se 
o tratamento endodôntico, com selamento convencional com guta-percha. $\mathrm{Na}$ radiografia realizada, observou-se bom processo de reparo e áreas de regeneração do ligamento periodontal (Figura 2). Nos acompanhamentos de 02,05 e 07 anos radiograficamente observou-se cicatrização do osso alveolar com reinserção do ligamento periodontal ao longo da raiz com formação de lâmina dura, sem evidência de reabsorção radicular inflamatória progressiva ou anquilose alvéolo dental (Figura 3). No acompanhamento de 13 anos radiograficamente observou-se reabsorção parcial por substituição. Paciente também havia iniciado tratamento ortodôntico 02 anos antes (Figura 4). De forma geral, após 13 anos do autotransplante paciente apresenta-se sem queixas álgicas e satisfeita com o tratamento. Elemento dentário sem mobilidade, mantendo a função oclusal e gengiva da região saudável.

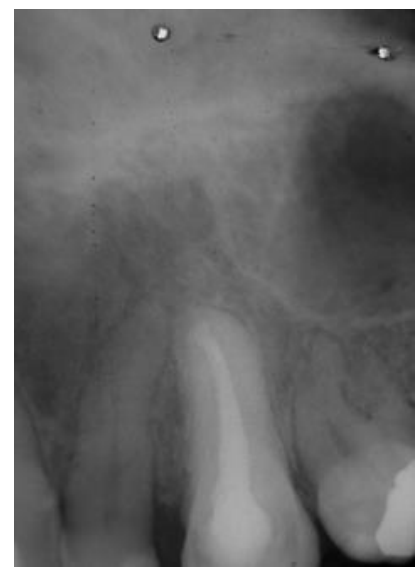

Figura 2: Tratamento endodôntico definitivo realizado 02 meses após o transplante. Notar bom reparo ósseo e áreas de reinserção do ligamento periodontal.

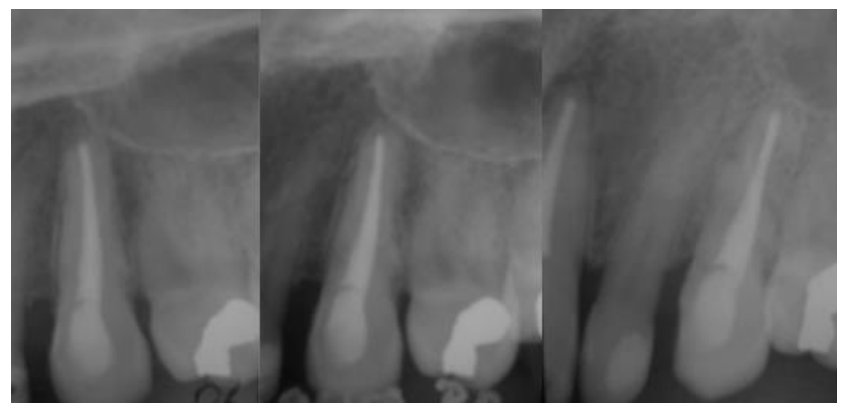

Figura 3: Pós-operatórios de 02 anos, 05 anos e 07 anos, respectivamente. Notar normalidade da cicatrização óssea alveolar e ligamento periodontal ao longo da raiz.

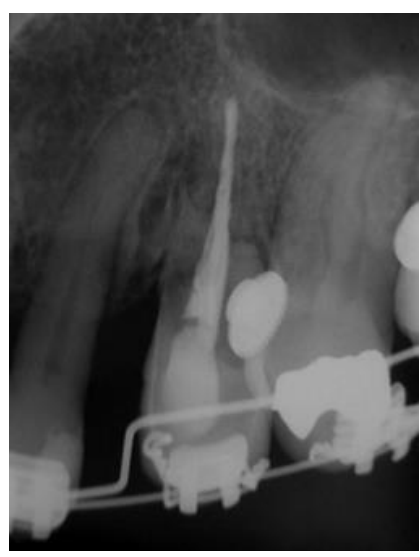

DISCUSSÃO

O transplante dental autógeno é uma opção para reabilitação em casos de perdas dentárias ou para elementos dentários inclusos, em que o espaço foi mantido ou não ${ }^{13}$. Nos casos de caninos impactados em que o espaço desse dente foi mantido pode-se optar pela confecção do alvéolo dentário, com a utilização de brocas, para adaptação do transplante dentário $^{3}$. O autotransplante dos caninos superiores deve ser considerado também devido ao valor estético e funcional desses dentes à arcada dentária ${ }^{14}$.

Em casos de caninos inclusos pode-se optar pela exodontia, com posterior reabilitação com implantes, ou então, o tracionamento ortodôntico associado à técnica cirúrgica ${ }^{5,15}$. Entretanto, a escolha pelo transplante autógeno, como suportado na literatura, pode funcionar como opção reabilitadora mais econômica e tempo efetiva ${ }^{16-18}$, além da indicação para os elementos inclusos que estão em posição não favorável ao tracionamento ${ }^{13}$. Pode ser considerado uma contraindicação para utilização deste método a dificuldade técnica para realização do procedimento ${ }^{19}$.

Conci et al. ${ }^{1}$ estabeleceram 17 anos como limite de idade máximo para autotransplantes dentários, e de preferência dentes com 2/3 da raiz formada. No caso relatado a paciente tinha 17 anos, porém, o elemento dentário já se encontrava com rizogênese completa.

O alvéolo receptor do transplante dentário deve ter: uma condição de osso alveolar adequada ${ }^{20,21}$, um diâmetro compatível com 0 dente ${ }^{4}$ e se possível durante sua confecção deve ser mantido o máximo de osso alveolar $^{17}$. Em Guralnick ${ }^{15}$ em casos de perda da lâmina óssea vestibular, o mucoperiósteo pode se reinserir na superfície radicular do transplante, sendo rara a formação de bolsa ou recessão periodontal. A perda da tabua óssea vestibular, ou defeitos ósseos aquém do planejado na confecção do alvéolo $^{22}$ não contraindica o autotransplante, assim como neste relato de caso. A quantidade de osso removida durante a confecção de alvéolos receptores não influenciou a condição periodontal de dentes transplantados ${ }^{23}$.

Em casos de reimplante e transplante dentário tem-se como prioridade a preservação do ligamento periodontal, devendo-se evitar a manipulação da porção radicular do dente, aumentando-se assim, as possibilidades de reinserção dos tecidos de sustentação no sítio receptor dos elementos dentários ${ }^{2,4,24,25}$. Na 
apicectomia do caso apresentado a utilização do alicate de corte ortodôntico, ao invés alta de rotação e brocas cirúrgicas, permitiu a diminuição do tempo fora do alvéolo do dente à ser transplantado.

O período de cicatrização dos ligamentos periodontais, nos transplantes autógenos está relacionado com o tipo e o tempo de contenção utilizado ${ }^{26}$. No caso exposto foi utilizado uma contenção de amarria com fio de aço, durante 4 semanas. Período este aproximado ao necessário para cicatrização do ligamento alveolar, em casos onde se deseje esperar a remodelação do alvéolo o tempo de contenção pode ser estendido de 6 a 8 semanas ${ }^{1}$.

Para evitar danos mecânicos e químicos nos tecidos de cicatrização do elemento dentário transplantado é sugerido iniciar o tratamento endodôntico 4 semanas após a cirurgia $^{16}$. No relato apresentado o tratamento endodôntico começou após 15 dias do transplante, com a sua finalização após 40 dias. Foi relatado na literatura o início de tratamento endodôntico 3 semanas após o transplante, evidenciando sucesso, sem danos aos tecidos de cicatrização ou reabsorção radicular ${ }^{2}$. Contudo dentes onde o tratamento endodôntico é iniciado tardiamente, após 10 semanas ${ }^{27}$, apresentaram maiores porcentagens de reabsorção radicular ${ }^{22}$.

$$
\text { E descrito por Ahlberg et al. }{ }^{8} \text {, }
$$
Andreasen $^{28}$ e Patel ${ }^{20}$, que os caninos transplantados têm bom prognóstico com taxa de sobrevida entre $86 \%$ a $100 \%$ em cavidade oral. Neste relato de caso, 0 dente transplantado está viável há 13 anos, em função, sem mobilidade, sem alterações em tecido mole ou formação de bolsa periodontal, o que vai de encontro com esses trabalhos.

CONCLUSÃO

Pode-se concluir que o resultado do caso descrito foi aceitável, produzindo uma solução clínica, estética e social, durante pelo menos 13 anos. Mostrou que a técnica do autotransplante de canino incluso é um método com bom prognóstico, sendo uma alternativa para substituir o dente ausente, e preservou nesse período a estrutura óssea e o espaço, possibilitando outras formas de reabilitação futuras.

\section{REFERÊNCIAS}

1. Conci RA, Martins JRP, Colet D, Huber HA, Battistetti GD, Sinegalia AC et al. Transplante dental-relato de um caso clínico. RFO UOF. 2011;16(3):322-26.

2. Candeiro GT, Alencar-Júnior EA, Scarparo HC,
Furtado-Júnior JH, Gavini G, Caldeira CL. Eight-year follow-up of autogenous tooth transplantation involving multidisciplinary treatment. J Oral Sci. 2015;57(3):273-76.

3. Park JH, Tai K, Hayashi D. Tooth autotransplantation as a treatment option: a review. J Clin Pediatr Dent. 2010;35(2):129-35.

4. Jodas CRP, Rapoport A, Junqueira JLC, Baccarin LS, Moraees PC, Gati RAR et al. Transplante dental bilateral: relato de caso clínico com acompanhamento de 9 anos. Rev Assoc Paul Cir Dent. 2012;66(1):30-5.

5. Britto AM, Fraga CFF, Goursand D, Costa EN, Grossi E, Rocha Junior JF. Impactação de caninos superiores e suas consequências: relato de caso clínico. J Bras Ortodon Ortop Facial 2003; 8(48):453-59.

6. Gaetti-Jardim EC, Faria KM, Santiago-Junior JF, Gaetti-Jardim Junior E, Saad Neto M, Aranega $\mathrm{AM}$ et al. Condutas terapêuticas para caninos inclusos. UNOPAR Cient Ciênc Biol Saúde. 2012;14(1):51-6.

7. Simão TM, Crepaldi MV, Neves MJG, Yamate EM, Burger RC. Tracionamento ortodôntico de caninos superiores impactados por palatino. Rev FAIPE. 2017;2(1):29-40.

8. Ahlberg K, Bystedt $H$, Eliasson S, Odenrick L. Long-term evaluation of autotransplanted maxillary canines with completed root formation. Acta Odontol Scand. 1983;41(1): 23-31.

9. Berglund L, Kurol J, Kvint S. Orthodontic pretreatment prior to autotransplantation of palatally impacted maxillary canines: case reports on a new approach. Eur $\mathrm{J}$ Orthod. 1996;18(5):449-56.

10. Foreman PC, Barnes IE. Review of calcium hydroxide. Int Endod J. 1990;23(6):283-97.

11. Toledo R, Britto MLB, Pallotta RC, Nabeshima CK. Hidróxido de Cálcio e lodofórmio no tratamento endodôntico de dentes com Rizogênese Incompleta. IJD Int J Dent. 2010; 9(1):28-37.

12. Laskin DM. Cirurgia Bucal e Maxilofacial. Chicago: Quintessence; 1991.

13. Rocha GNP. Relação entre cirurgia e ortodontia autotransplantes dentários. Port. Implantol. 2002(1):24-6.

14. Saad Neto M, Carvalho ACP. Caninos inclusos I: etiologia, incidência e tratamento. Rev Odontol Aracatuba. 1983:19-34.

15. Guralnick WC. Autogenous and allogeneic transplantation of teeth. $\mathrm{J}$ Oral Surg. 1970;28(8):575-77.

16. Arikan F, Nizam N, Sonmez S. 5-year longitudinal study of survival rate and periodontal parameter changes at sites of maxillary canine autotransplantation. J Periodontol. 2008;79(4):595-602.

17. Reich PP. Autogenous transplantation of 
maxillary and mandibular molars. $\mathrm{J}$ Oral Maxillofac Surg. 2008;66(11):2314-17.

18. Tsukiboshi M. Autotransplantation of teeth: requirements for predictable success. Dent Traumatol. 2002;18(4):157-80.

19. Silva Filho OG, Fugio N, Capelozza Filho L, Cavassan AO. Irrupçäo ectópica dos caninos permanentes superiores: soluçöes terapêuticas. Ortodontia;27(3):50-66,

20. Patel S, Fanshawe T, Bister D, Cobourne MT. Survival and success of maxillary canine autotransplantation: a retrospective investigation. Eur J Orthod. 2011;33(3): 298-304.

21. Kafourou V, Tong HJ, Day P, Houghton N, Spencer RJ, Duggal M. Outcomes and prognostic factors that influence the success of tooth autotransplantation in children and adolescents. Dent Traumatol. 2017;33(5): 393-99.

22. Machado LA, do Nascimento RR, Ferreira DM, Mattos CT, Vilella OV. Long-term prognosis of tooth autotransplantation: a systematic review and meta-analysis. Int J Oral Maxillofac Surg. 2016;45(5):610-17.

23. Sagne S, Thilander B. Transalveolar transplantation of maxillary canines. A critical evaluation of a clinical procedure. Acta Odontol Scand. 1997;55(1):1-8.

24. Silva Junior EZ, Silva TMV, Esteves GB, Rolim HSF, Dourado ACAG. Prognóstico e tratamento da avulsão dentária: relato de caso. Rev cir traumatol buco-maxilo-fac. 2015;15(3):39-42.

25. Victorino FR, Gottardo VD, Zadetto Junior R, Moreschi E, Zamponi M, Trento CL. Reimplante dentário para 0 tratamento de Avulsão Dentária: relato de caso clínico. Rev assoc paul cir dent. 2013;67(3):202-6.

26. Bauss O, Schwestka-Polly R, Schilke R, Kiliaridis $S$. Effect of different splinting methods and fixation periods on root development of autotransplanted immature third molars. J Oral Maxillofac Surg. 2005;63(3):304-10.

27. Sagne S, Lennartsson B, Thilander B. Transalveolar transplantation of maxillary canines. An alternative to orthodontic treatment in adult patients. Am J Orthod Dentofacial Orthop. 1986;90(2):149-57.

28. Andreasen JO. Atlas de reimplante e transplante de dentes. São Paulo: Panamericana; 1994.

\section{CONFLITO DE INTERESSES}

Os autores declaram não haver conflitos de interesse

\section{AUTOR PARA CORRESPONDÊNCIA}

\section{Ellen Cristina Gaetti Jardim}

Universidade Federal de Mato Grosso do Sul - UFMS

Cidade Universitária, $\mathrm{s} / \mathrm{n}$ Universitário

79070-900 Campo Grande - MS, Brasil

(67) 3345-7682

ellen.jardim@ufms.br 